\title{
Norepinephrine enhances cell viability and invasion, and inhibits apoptosis of pancreatic cancer cells in a Notch-1-dependent manner
}

\author{
WEIKUN QIAN $^{1 *}$, SHIFANG LV ${ }^{2 *}$, JIE LI $^{1}, \mathrm{KE} \mathrm{CHEN}^{1}$, ZHENGDONG JIANG $^{1}$, LIANG CHENG $^{1}$, \\ CANCAN ZHOU $^{1}$, BIN YAN ${ }^{1}$, JUNYU CAO $^{1}$, QINGYONG MA ${ }^{1}$ and WANXING DUAN ${ }^{1}$ \\ ${ }^{1}$ Department of Hepatobiliary Surgery, The First Affiliated Hospital, Xi'an Jiaotong University, Xi'an, Shaanxi 710061; \\ ${ }^{2}$ Department of Abdominal Surgery, Tumor Hospital of Linyi City, Linyi, Shandong 276001, P.R. China
}

Received January 9, 2018; Accepted August 6, 2018

DOI: 10.3892/or.2018.6696

\begin{abstract}
Pancreatic ductal adenocarcinoma (PDAC) is one of the most aggressive types of cancer, which is associated with a poor prognosis due to complexities in prevention, early diagnosis and effective treatment. The lack of understanding regarding its induction and specific pro-cancer mechanisms may contribute to its poor prognosis. The Notch-1 pathway is widely considered to be a critical tumor-promoting factor in PDAC. Previous studies have indicated that chronic psychological stress may promote the development of PDAC partially via the main downstream stress hormone, norepinephrine (NE); however, to the best of our knowledge, the role of the Notch-1 pathway in this process has not been studied. Therefore, the present study aimed to explore this process. The expression levels of Notch-1 pathway-associated molecules were measured in response to NE using reverse transcription-quantitative polymerase chain reaction and western blotting. Alongside NE treatment, two Notch-1 pathway blockers, Notch-1-specific small interfering (si)RNA and DAPT (an inhibitor of the Notch-1 pathway), were used to explore the relationship between NE and the Notch-1 pathway in the development of pancreatic cell malignant biological behaviors, including cell viability, apoptosis and cell invasion. The results demonstrated that treatment with NE enhanced cell viability and invasion, and inhibited apoptosis of PDAC cells; however, these effects were suppressed following treatment
\end{abstract}

Correspondence to: Dr Wanxing Duan or Professor Qingyong Ma, Department of Hepatobiliary Surgery, The First Affiliated Hospital, Xi'an Jiaotong University, 277 West Yanta Road, Xi'an, Shaanxi 710061, P.R. China

E-mail: 15339184808@163.com

E-mail: qyma56@xjtu.edu.cn

${ }^{*}$ Contributed equally

Key words: pancreatic ductal adenocarcinoma, norepinephrine, Notch-1 pathway, malignant biological behavior with Notch-1-specific siRNA and DAPT. In conclusion, NE may enhance the malignant biological behaviors of PDAC via activating the Notch-1 pathway.

\section{Introduction}

Pancreatic ductal adenocarcinoma (PDAC) has a 5-year survival rate of $<8 \%$ (1), and is characterized by a highly aggressive nature and poor response to clinical treatment. Only a small percentage of patients are able to receive radical resection, which is the only curative treatment option, and/or adjuvant chemotherapy with agents such as gemcitabine and the oral fluoropyrimidine derivative $S-1$, or other advanced therapeutic strategies. Among patients who undergo radical resection, the 5-year survival rate is still only $25 \%$ (2). At present, little is known about the factors that contribute to the initiation and progression of PDAC, and its specific underlying mechanisms.

Chronic psychological stress is considered to be a powerful tumor promoter in numerous types of cancer, including PDAC, via inducing activation of the hypothalamic pituitary adrenal (HPA) axis and/or the sympathetic nervous system (SNS) $(3,4)$. Norepinephrine (NE) is a major stress hormone, which serves a vital role in the chronic psychological stress that may induce tumor progression. According to Lara et al (5), the concentration of $\mathrm{NE}$ can be $\leq 10 \mu \mathrm{M}$ in the tumor microenvironment, and NE may promote the proliferation, invasion, migration and malignant biological behaviors of PDAC via activation of the $\beta 2$-adrenergic receptor ( $\beta 2-\mathrm{AR})$ in vitro and in vivo (6-9). However, these studies did not elucidate the possible downstream mechanisms; therefore, additional research is required.

The Notch-1 pathway is involved in several physical and pathological biological processes, including cancer (10-15). Notably, Notch is essential for embryonic development of the pancreas and is involved in the plasticity of adult exocrine cells (16-18); in addition, abnormal activation of the Notch-1 pathway is correlated with the initiation and progression of PDAC $(10,11,19)$. Previous studies reported that chronic stress inhibits differentiation, and maintains the stem cell state of hematopoietic stem cells via activating the Notch-1 pathway $(20,21)$. Furthermore, NE may promote angiogenesis 
in breast cancer via upregulation of the Notch pathway molecule Jagged-1 (22). These studies suggest that chronic stress, NE and the Notch-1 pathway may have an interactive relationship in PDAC. The present study hypothesized that the stress hormone NE may activate the Notch-1 pathway in PDAC, thus contributing to its malignant biological behaviors.

\section{Materials and methods}

Cell culture and reagents. Human PDAC cell lines (AsPc-1, BxPc-3, Panc-1, HPAC, Mia PaCa-2 and SW1990) were purchased from the Chinese Academy of Sciences Cell Bank of Type Culture Collection (Shanghai, China). Panc-1 and Mia PaCa-2 cells were cultured in Dulbecco's modified Eagle's medium (DMEM; HyClone; GE Healthcare Life Sciences, Logan, UT, USA), HPAC and BxPc-3 cells were cultured in RPMI-1640 (DMEM; HyClone; GE Healthcare Life Sciences); both media were supplemented with $10 \%$ fetal bovine serum (FBS; HyClone; GE Healthcare Life Sciences, Logan, UT, USA) and $1 \%$ penicillin-streptomycin. AsPc-1 cells were cultured in RPMI-1640 supplemented with $20 \%$ FBS and $1 \%$ penicillin-streptomycin. SW1990 cells were cultured in L-15 Leibovitz media (HyClone; GE Healthcare Life Sciences) supplemented with $10 \%$ FBS and $1 \%$ penicillin-streptomycin. The cells were cultured under standard conditions in an atmosphere containing $5 \% \mathrm{CO}_{2}$ at $37^{\circ} \mathrm{C}$. To determine the optimal concentration and duration of treatment, BxPc-3 and Panc-1 cells were treated with $\mathrm{NE}$ at various concentrations $(0,0.1,1$ or $10 \mu \mathrm{M})$ for $24 \mathrm{~h}$, or were treated with a fixed concentration of $\mathrm{NE}(10 \mu \mathrm{M})$ for various durations $(0,12,24$ or $48 \mathrm{~h})$. Panc-1 and BxPc-3 cells were separated into the following four groups for each assay: Negative control (medium only), NE (10 $\mu \mathrm{M})$, $\mathrm{NE}(10 \mu \mathrm{M})+\mathrm{DAPT}(50 \mu \mathrm{M})$ and $\mathrm{NE}(10 \mu \mathrm{M})+$ small interfering (si)RNA-Notch-1 at $37^{\circ} \mathrm{C}$ for $48 \mathrm{~h}$. Subsequently, these cells underwent $\mathrm{mRNA}$ /protein extraction and various assays were conducted. NE and DAPT (Notch-1 pathway inhibitor) were purchased from Sigma-Aldrich; Merck KGaA (Darmstadt, Germany).

The following antibodies were used in the present study: Anti- $\beta 2-A R$ (cat. no. sc-569, 1:200; Santa Cruz Biotechnology, Inc., Dallas, TX, USA), anti-Notch-1 (cat.no. 3608, 1:1,000; Cell Signaling Technology, Inc., Danvers, MA, USA), anti-Jagged-1 (cat. no. ab109536, 1:1,000; Abcam, Cambridge, MA, USA), anti- $\beta$-actin (cat. no. 3700, 1:1,000; Cell Signaling Technology, Inc.), anti-recombination signal binding protein for immunoglobulin $\kappa \mathrm{J}$ region (RBP-J $\kappa$; cat. no. ab180588, 1:1,000; Abcam), anti-Hes-1 (cat. no. ab108937, 1:1,000; Abcam), anti-matrix metalloproteinase (MMP)-2 (cat. no. ab97779, 1:1,000; Abcam), and anti-MMP-9 (cat. no. ab38898, 1:1,000; Abcam).

siRNA transfection. Notch-1-specific siRNA (si-Notch-1; Notch-1 siRNA-780; sense 5'-GUCCAGGAAACAACUGCA ATT-3' and antisense 5'-UUGCAGUUGUUUCCUGGACT-3') and a negative control siRNA (sense, 5'-UUCUCCGAACGU GUCACGUTT-3' and antisense, 5'-ACGUGACACGUUCGG AGAATT-3') were purchased from Shanghai GenePharma Co. Ltd. (Shanghai, China). Cells $\left(0.5 \times 10^{4}\right)$ were seeded in 6-well plates and were transfected at $37^{\circ} \mathrm{C}$ with $100 \mathrm{nM}$ siRNAs using Lipofectamine RNAi MAX Reagent (Invitrogen; Thermo
Fisher Scientific, Inc., Waltham, MA, USA), according to the manufacturer's protocol. Cells were used in the subsequent experiments $24 \mathrm{~h}$ post-transfection.

Reverse transcription-quantitative polymerase chain reaction $(R T-q P C R)$. Total RNA was extracted using the Fastgen 200 RNA isolation system (Fastgen, Shanghai, China), according to the manufacturer's protocol. A Prime Script RT reagent kit (Takara Biotechnology Co., Ltd., Dalian, China) was used to reverse transcribe total RNA into cDNA, according to the manufacturer's protocol. qPCR was conducted using an iQ5 Multicolor Real-Time PCR Detection system (Bio-Rad Laboratories, Inc., Hercules, CA, USA) and a SYBR Green PCR kit (Takara Biotechnology Co., Ltd.). The following PCR program was used: Denaturation at $94^{\circ} \mathrm{C}$ for $5 \mathrm{~min}$, followed by 35 cycles consisting of denaturation at $94^{\circ} \mathrm{C}$ for $30 \mathrm{sec}$, annealing at $60^{\circ} \mathrm{C}$ for $30 \mathrm{sec}$ and extension at $72^{\circ} \mathrm{C}$ for $45 \mathrm{sec}$; finally, the samples were incubated at $72^{\circ} \mathrm{C}$ for $5 \mathrm{~min}$ and then maintained at $4^{\circ} \mathrm{C}$. The specificity of the amplified PCR products was evaluated by melting curve analysis. The comparative $\mathrm{Cq}$ method (23), with $\beta$-actin as the normalization control, was used to assess the expression level of each target gene, as previously described (24). The PCR primer sequences used were as follows: Jagged-1, forward TTG GTTAATGGTTATCGCTGTATC, and reverse GCAGTT CTTGCCCTCATAGTCC; Notch-1, forward GGCACTTTC TGTGAGGAGGA, and reverse GCAGTCAGGCGTGTT GTTCT; RBP-Jк, forward GACTCAGACAAGCGAAAG CA, and reverse GTCGATTAAACAGAGCCACC; Hes-1, forward TAGCTCGCGGCATTCCAAG, and reverse AAG CGGGTCACCTCGTTCA; MMP-2, forward GATGATGCC TTTGCTCGTGC, and reverse CAAAGGGGTATCCATCGC CA; MMP-9, forward TCCACCCTTGTGCTCTTCCCT, and reverse CTGCCACCCGAGTGTAACCA; and $\beta$-actin forward GACTTAGTTGCGTTACACCCTTTCT, and reverse GAACGGTGAAGGTGACAGCAGT. The housekeeping gene $\beta$-actin was used as an internal control.

Western blot analysis. Whole-cell lysates of Panc-1 and BxPC-3 cells were prepared using radioimmunoprecipitation assay buffer (Beyotime Institute of Biotechnology, Haimen, China) according to the manufacturer's protocol. The protein concentration was determined using a bicinchoninic acid protein assay kit (Pierce; Thermo Fisher Scientific, Inc.). The protein lysates were resolved on a $10 \%$ polyacrylamide gel with a 5\% stacking gel. The proteins were subsequently transferred to polyvinylidene difluoride membranes. The membranes were blocked for $2 \mathrm{~h}$ in Tris-buffered saline- $0.1 \%$ (vol/vol) Tween-20 (TBST) containing $10 \%$ (wt/vol) nonfat dry milk powder at room temperature and were then incubated with primary antibodies overnight at $4^{\circ} \mathrm{C}$. Following incubation with goat anti-rabbit immunoglobulin $\mathrm{G}(\mathrm{IgG})$-horseradish peroxidase (HRP) (cat. no. sc-2004, 1:10,000) and goat anti-mouse IgG-HRP (cat. no. sc-2005, 1:10,000) secondary antibodies (Santa Cruz Biotechnology, Inc.), for $2 \mathrm{~h}$ at room temperature, the membranes were washed with TBST, and the immunocomplexes were detected using an enhanced chemiluminescence kit (EMD Millipore, Billerica, MA, USA) and the Molecular Imager ChemiDoc XRS system (Bio-Rad Laboratories, Inc.). $\beta$-actin was used as the internal loading control. 


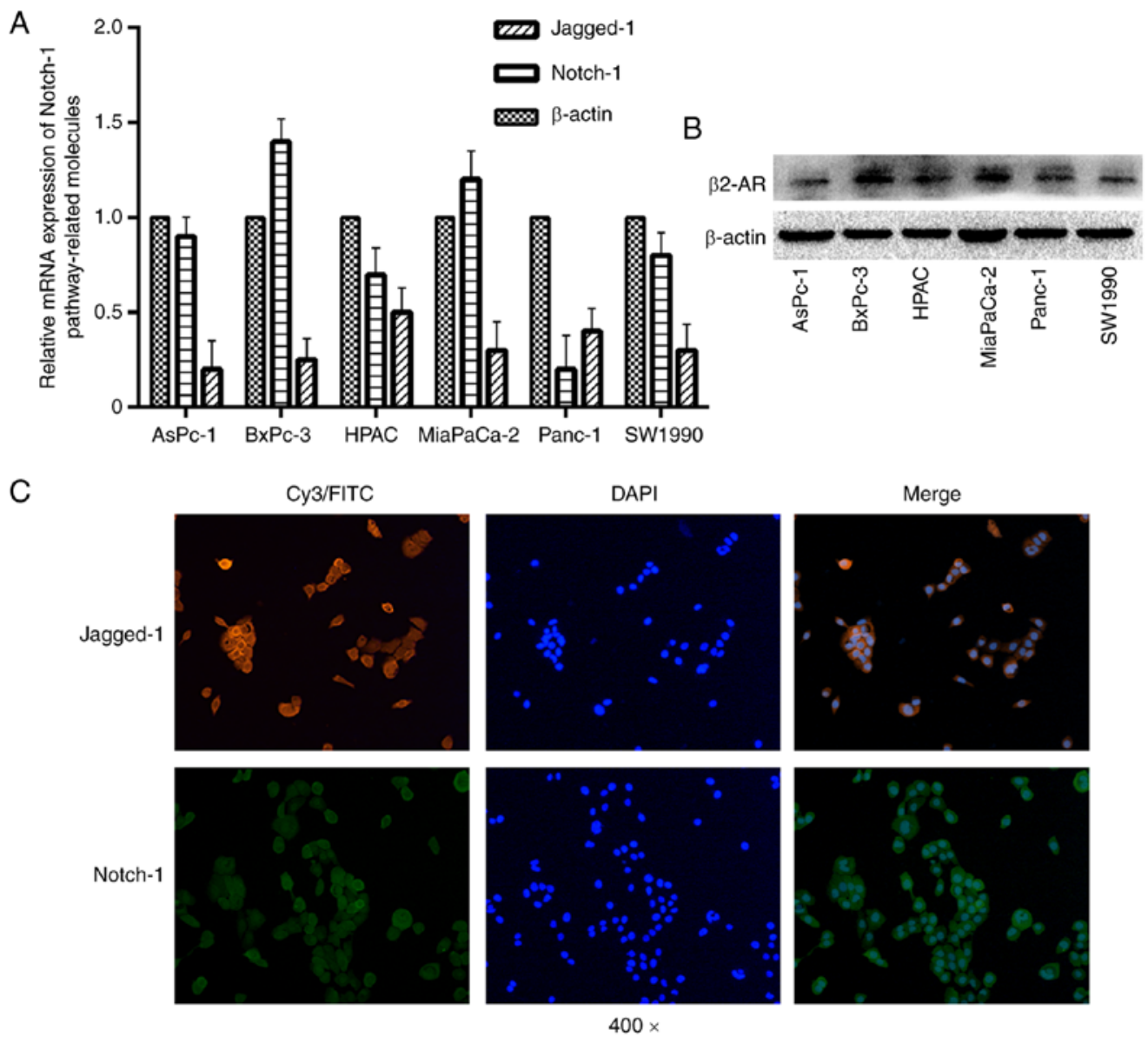

Figure 1. Expression and location of Notch-1 pathway-associated molecules in pancreatic ductal adenocarcinoma cells. (A) Relative mRNA expression levels of Notch-1 pathway-associated molecules in six pancreatic cancer cell lines. (B) Relative protein expression levels of $\beta 2$-AR in six pancreatic cancer cell lines. (C) Immunofluorescence analyses (magnification, $\mathrm{x} 400$ ) were conducted to detect the expression and location of Notch-1 pathway-associated molecules (Notch-1 and Jagged-1) in BxPC-3 cells. $\beta 2$-AR, $\beta 2$-adrenergic receptor; FITC, fluorescein isothiocyanate.

Immunofluorescence analysis. After applying the aforementioned intervention strategies, the cancer cells were fixed in $4 \%$ formaldehyde diluted in PBS for $20 \mathrm{~min}$ at room temperature. After permeabilization with $0.3 \%$ Triton X-100, the cells were treated with blocking buffer [5\% bovine serum albumin (Sigma-Aldrich; Merck KGaA) in PBS] for $1 \mathrm{~h}$, and then incubated with the primary antibodies at $4^{\circ} \mathrm{C}$ overnight. The cells were then incubated with Alexa Fluor 488-conjugated goat anti-rabbit IgG (green) secondary antibodies (cat. no. 111-545-003, 1:200; Jackson Immunoresearch Laboratories, Inc., West Grove, PA, USA) at room temperature for $30 \mathrm{~min}$, and the nuclei were stained with DAPI. Images were pseudo-colored using a Zeiss Instruments confocal microscope (Zeiss GmbH, Jena, Germany).

MTT assay. Cell viability was analyzed using an MTT assay according to a previously described method (25). Cancer cells were seeded in 96-well tissue culture plates at a density of 5,000-10,000 cells/well $24 \mathrm{~h}$ prior to serum starvation. After serum starvation for $24 \mathrm{~h}$, cells were cultured in medium and were treated with the aforementioned intervention strategies. After 12, 24 or $48 \mathrm{~h}$, the medium was removed, and MTT reagent was added to each well and incubated at $37^{\circ} \mathrm{C}$ for $4 \mathrm{~h}$. Subsequently, $150 \mu \mathrm{l}$ dimethyl sulfoxide was added to each well and the cells were incubated in the dark for $10 \mathrm{~min}$ at room temperature. Optical density (OD) values were measured at $490 \mathrm{~nm}$ using a microplate reader (BioTek Instruments, Inc., Winooski, VT, USA). Cell viability rate was defined as follows: OD (sample well)/OD (control well).

Apoptosis assay. Cell apoptosis was assessed by flow cytometry using an Annexin V-fluorescein isothiocyanate (FITC)/propidium iodide (PI) apoptosis detection kit (BD Biosciences, San Diego, CA, USA), according to the manufacturer's protocol, as previously described (26). Briefly, cancer cells were seeded into 6-well plates at a density of $2 \times 10^{5}$ cells/well, and after being serum-starved overnight, the cells were treated with the aforementioned intervention strategies for 48 h. Subsequently, the cells were trypsinized, washed with PBS and stained with Annexin V and PI. The percentage of apoptotic cells was quantified by flow cytometry using a FACSCalibur (BD Biosciences) instrument. Samples were analyzed and the percentage of apoptotic cells was evaluated.

Cell invasion assay. A Matrigel invasion assay was performed as previously described (27), in order to assess the invasive ability of PDAC cells. Briefly, the upper chambers of the wells were coated with Matrigel (BD Biosciences). Following treatment with the aforementioned intervention strategies for $48 \mathrm{~h}$, the cancer cells $\left(5 \times 10^{5}\right)$ were suspended in serum-free medium 

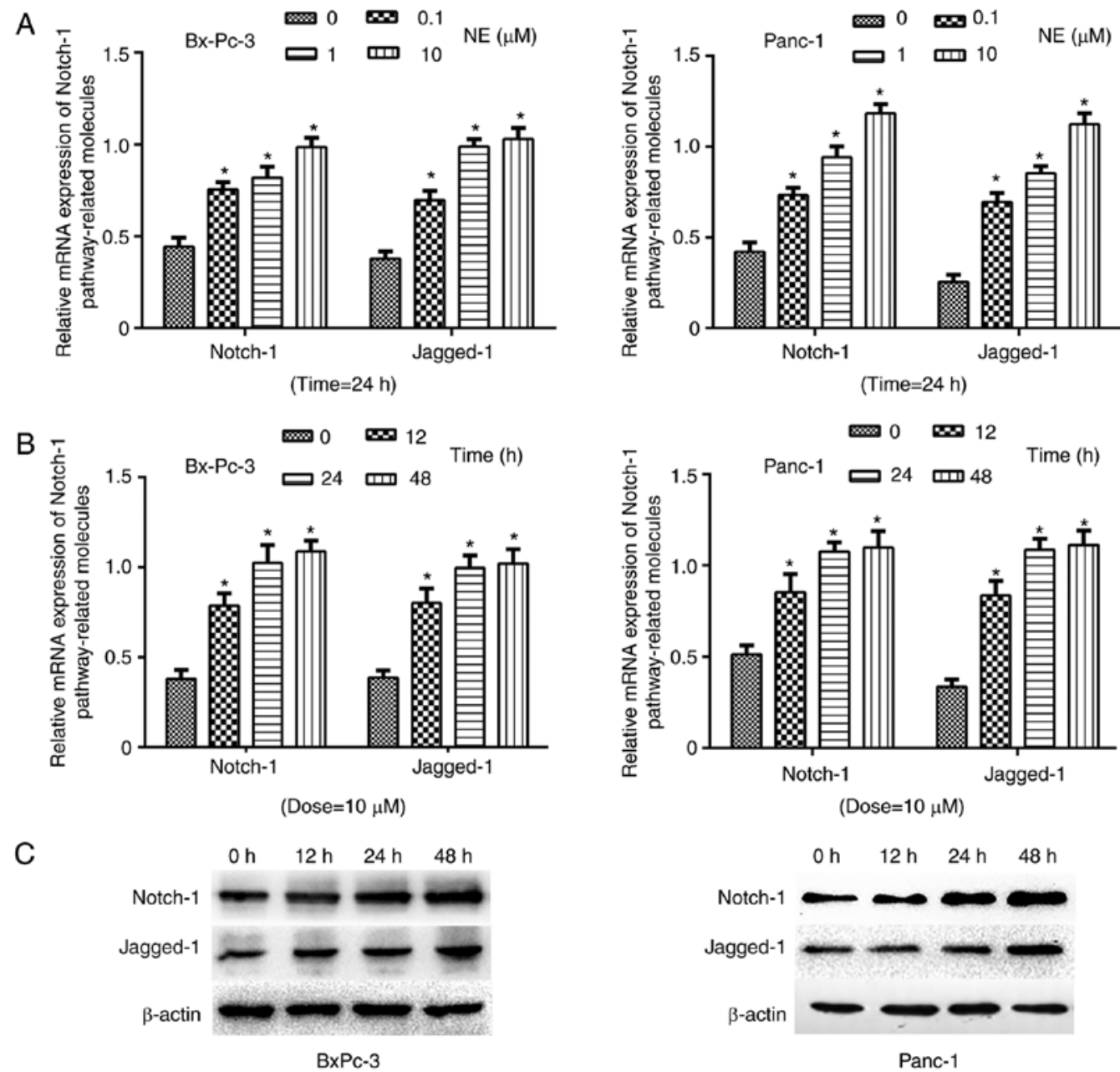

Figure 2. NE promotes the expression of Notch-1 pathway-associated genes in pancreatic ductal adenocarcinoma cells. (A) Relative mRNA expression levels of Notch-1 pathway-associated molecules (Notch-1 and Jagged-1) were detected in BxPC-3 and Panc-1 cells following exposure to increasing doses of NE $(0,0.1,1$ or $10 \mu \mathrm{M})$ by RT-qPCR. $\beta$-actin used as an internal control. "P<0.05 vs. the control group. (B) Relative mRNA expression levels of Notch-1 pathway-associated molecules were detected in BxPc-3 and Panc-1 cells following treatment with $1010 \mu \mathrm{M}$ NE for various durations $(0,12,24$ or $48 \mathrm{~h})$ by RT-qPCR. $\beta$-actin used as an internal control. "P<0.05 vs. the control group. (C) Protein expression levels of Notch-1 and Jagged-1 were detected in BxPc-3 and Panc-1 cells following treatment with $10 \mu \mathrm{M}$ NE for various durations $(0,12,24$ or 48 h) by western blotting. NE, norepinephrine; RT-qPCR, reverse transcription-quantitative polymerase chain reaction.

and seeded into the upper chamber. Cells were allowed to migrate toward media (DMEM/RPMI-1640) supplemented with $10 \% \mathrm{FBS}$ in the lower chamber at $37^{\circ} \mathrm{C}$ for $24 \mathrm{~h}$. The media were aspirated from the inside of the insert, and the non-invasive cells on the upper side were removed using a cotton swab. The membrane of the chamber was then fixed with $4 \%$ paraformaldehyde for $15 \mathrm{~min}$ at room temperature and stained with $0.1 \%$ crystal violet for $15 \mathrm{~min}$ at $37^{\circ} \mathrm{C}$. The number of invading cells was quantified by counting the stained cells under a light microscope (Nikon Corporation, Tokyo, Japan).

Statistical analysis. Each experiment was performed at least three times. Data are presented as the means \pm standard deviation. Using GraphPad Prism version 6.0 software (GraphPad Software, Inc., La Jolla, CA, USA), differences among groups were assessed by one-way analysis of variance followed by Dunnett's test for multiple comparisons. All tests were two sided, $\mathrm{P}<0.05$ was considered to indicate a statistically significant difference.

\section{Results}

Expression and location of Notch-1 pathway-associated molecules in PDAC cells. The present study detected the expression levels of Notch-1 pathway-associated molecules in the pancreatic cell lines. The results demonstrated that the expression levels of Notch-1 and its ligand Jagged-1 were different in all of the cell lines tested (Fig. 1A). $\beta 2$-AR is a corresponding receptor of NE, which has been reported to be upregulated in pancreatic cancer tissue (28). The present study detected the protein expression levels of $\beta 2$-AR in six pancreatic cancer cell lines; the results revealed that $\beta 2-\mathrm{AR}$ expression was different in all cell lines analyzed (Fig. 1B). The present study selected two PDAC cell lines, BxPC-3 and Panc-1, for subsequent experiments and used them to determine the location of Notch-1 and Jagged-1. In BxPC-3 and Panc-1 cells (data not shown), Notch-1 and Jagged-1 were predominantly located on the cytomembrane, rather than in the cytoplasm or nucleus (Fig. 1C), which is consistent with the previous findings that the Notch pathway can be activated 
A

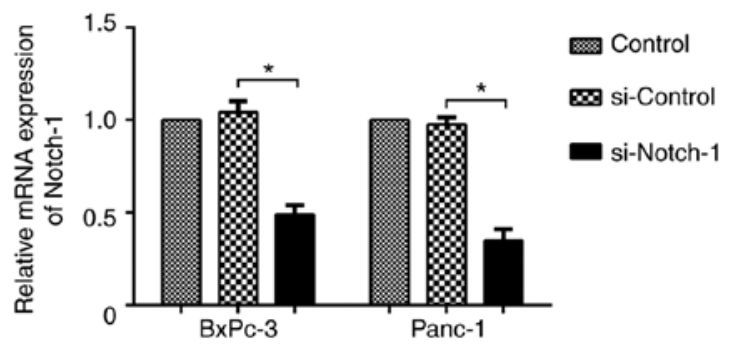

C
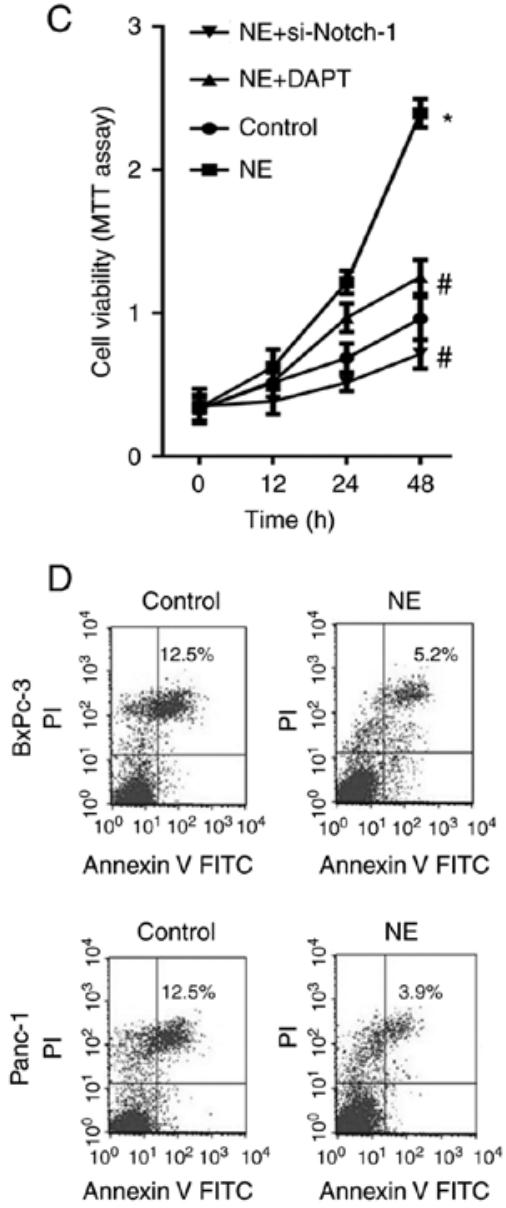
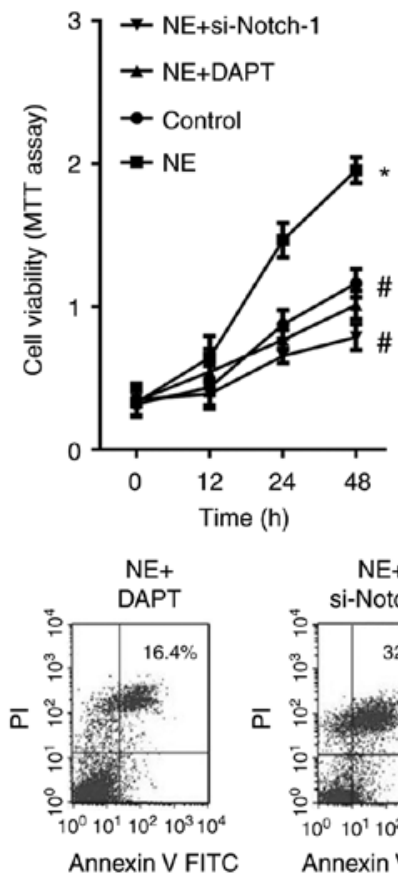

$\mathrm{NE}+$

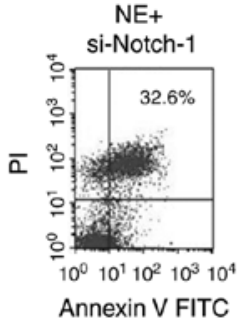

$\mathrm{NE}+$

si-Notch-1
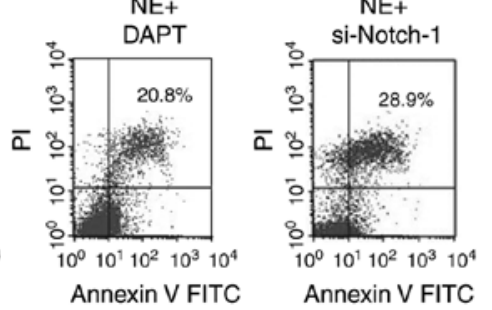

B

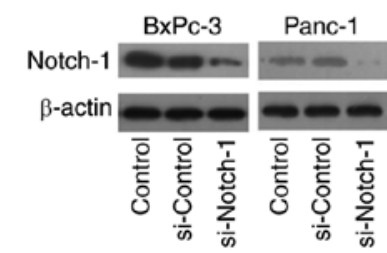

E

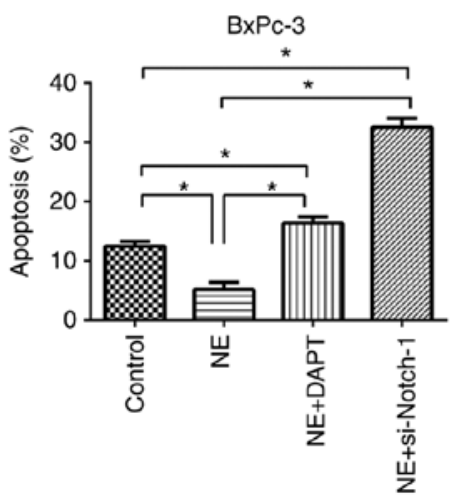

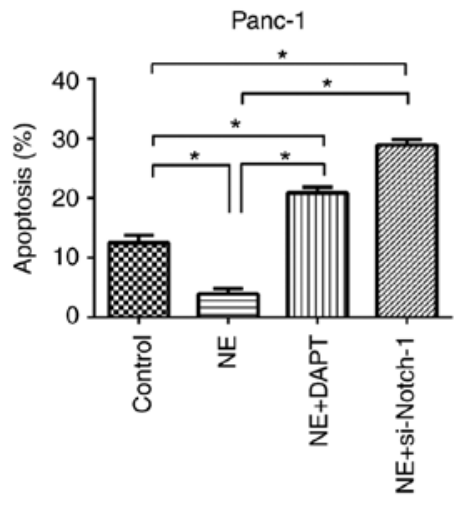

Figure 3. NE enhances cell viability and inhibits apoptosis of pancreatic ductal adenocarcinoma cells via activating the Notch-1 pathway. The silencing effects of si-Notch-1 on Notch-1 (A) mRNA and (B) protein expression were confirmed by reverse transcription-quantitative polymerase chain reaction and western blotting, respectively. (C) Viability of BxPC-3 and Panc-1 cells in the control, NE, NE+DAPT and NE+ si-Notch-1 groups, as determined using an MTT assay. ${ }^{\text {P }}<0.05$, vs. the control group; ${ }^{\#} \mathrm{P}<0.05$, vs. the NE group. (D and E) Apoptosis rate of BxPC-3 and Panc-1 cells in the control, NE, NE+DAPT and $\mathrm{NE}+$ si-Notch-1 groups. ${ }^{*} \mathrm{P}<0.05$ as indicated. NE, norepinephrine; si, small interfering RNA.

through various ligand-receptor interactions, such as the interaction between receptor Notch-1 and its ligand Jagged-1 (10). In conclusion, the Notch-1 pathway may be involved in the development of PDAC in vitro.

NE promotes the expression of Notch-1 pathway-associated genes in PDAC cells. To explore the effects of NE on Notch-1 pathway-associated gene expression in PDAC cells, the cells were treated with $\mathrm{NE}$ at various concentrations $(0,0.1,1$ or $10 \mu \mathrm{M})$. After $24 \mathrm{~h}$, Notch-1 pathway-associated gene expression was detected by RT-qPCR. The results demonstrated that as the concentration of NE increased, the expression levels of Notch-1 pathway-associated genes (Notch-1 and Jagged-1) were elevated in BxPC-3 and Panc-1 cells (Fig. 2A). Furthermore, PDAC cells were treated with $10 \mu \mathrm{M}$ NE for various durations $(0,12,24$ or 48 h). Subsequently, RT-qPCR and western blotting indicated that the expression levels of Notch-1 and Jagged-1 were increased as treatment duration increased (Fig. 2B and C). These findings indicated that NE may activate the Notch-1 pathway in PDAC cells, which is consistent with the hypothesis that NE may promote the progression of PDAC via activating the Notch-1 pathway.

NE enhances cell viability and inhibits apoptosis of PDAC cells via activation of the Notch-1 pathway. To further explore whether the Notch-1 pathway mediated the tumor-promoting effects of NE on PDAC, si-Notch-1 and DAPT were used to suppress the Notch-1 pathway in PDAC cells. As shown in Fig. 3A and B, si-Notch-1 effectively inhibited Notch-1 expression at both the mRNA and protein levels. To explore the role of 

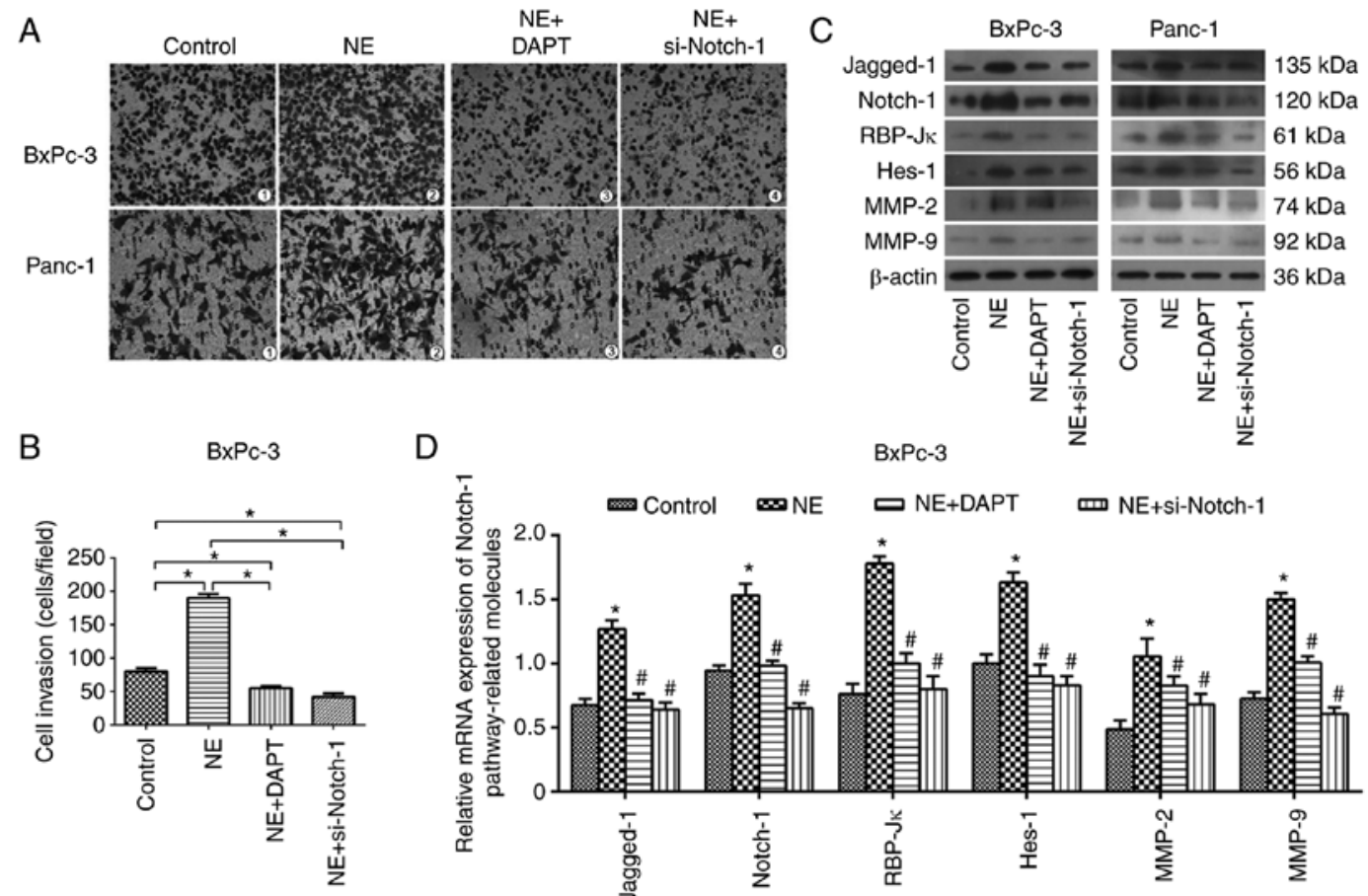

$\mathrm{D}$
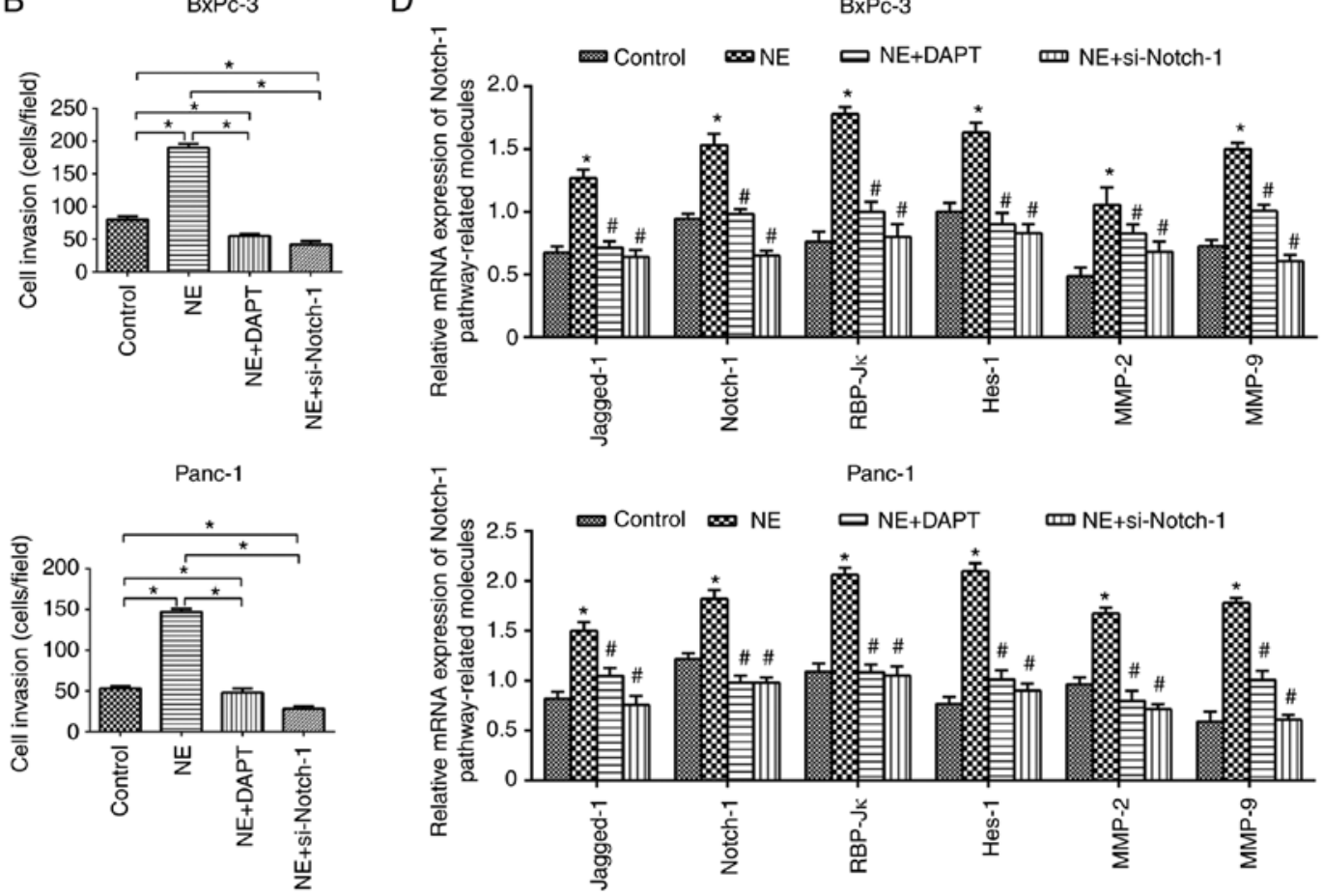

Figure 4. NE enhances the invasive ability of pancreatic ductal adenocarcinoma cells via activating the Notch-1 pathway. (A and B) Invasive ability of BxPC-3 and Panc-1 cells in the control, NE, NE+DAPT and NE+si-Notch-1 groups, as determined using a Transwell assay (magnification, $x 100$ ). Relative expression levels of Notch-1 pathway-associated and invasion-associated molecules at the (C) protein and (D) mRNA levels in BxPC-3 and Panc-1 cells in the control, $\mathrm{NE}, \mathrm{NE}+\mathrm{DAPT}$ and $\mathrm{NE}+$ si-Notch-1 groups. ${ }^{*} \mathrm{P}<0.05$ vs. the control group; ${ }^{*} \mathrm{P}<0.05$ vs. the NE group. MMP, matrix metalloproteinase; NE, norepinephrine; RBP-J $\kappa$, recombination signal binding protein for immunoglobulin $\kappa \mathrm{J}$ region; si, small interfering RNA.

Notch-1 in NE-mediated effects on cell viability and apoptosis of PDAC cells, cells were treated with DAPT or si-Notch-1. As shown in Fig. 3C, NE treatment alone significantly enhanced the viability of PDAC cells; however, this effect was abolished by si-Notch-1 and DAPT. Similarly, an apoptosis assay indicated that NE inhibited apoptosis of PDAC cells, whereas this effect was suppressed following inhibition of the Notch-1 pathway (Fig. 3D and E). These findings indicated that NE enhanced cell viability and inhibited apoptosis of PDAC cells via activation of the Notch-1 pathway.

$N E$ enhances the invasive ability of PDAC cells via activation of the Notch-1 pathway. The present study confirmed that NE may promote PDAC cell viability and inhibit apoptosis; therefore, the effects of NE on the invasive ability of PDAC cells were subsequently investigated using a Transwell assay. The results demonstrated that NE promoted the invasive ability of BxPC-3 and Panc-1 cells, whereas these effects could be blocked by si-Notch-1 and DAPT (Fig. 4A and B). Furthermore, it was revealed that NE upregulated the
mRNA and protein expression levels of critical Notch-1 pathway-associated and -targeted factors, such as Notch-1, Jagged-1, RBP-Jא (a mammalian CSL protein) and Hes-1, and invasion-associated molecules, including MMP-2 and MMP-9; however, these effects were reversed following inhibition of the Notch-1 pathway (Fig. 4C and D), which means NE enhanced the invasive ability of PDAC cells via activating the Notch-1 pathway.

\section{Discussion}

Complexities in the prevention, diagnosis and treatment of PDAC may be partly due to the fact that little is currently known regarding the factors involved in PDAC progression and its specific mechanisms. However, the cancer-promoting effects of chronic psychological stress, and its major downstream stress hormone NE, are widely known $(3,29,30)$.

Chronic psychological stress can influence various physical and pathological biological processes, and it has been reported to potentially initiate the progression of cancer 


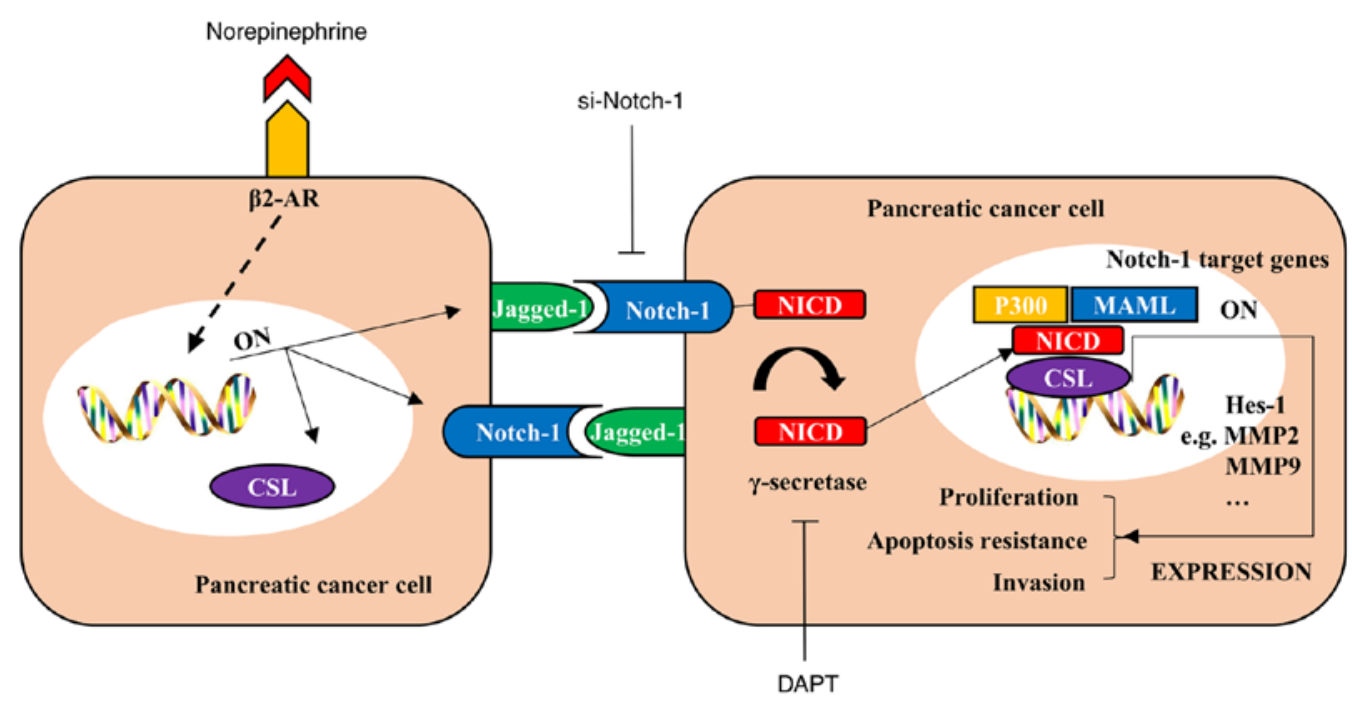

Figure 5. NE promotes malignant biological behaviors of pancreatic cancer cells in a Notch-1-dependent manner. NE binds to its receptor $\beta 2$-AR and activates downstream signaling, which elevates the expression of critical Notch-1 pathway-associated molecules, such as Jagged-1, Notch-1 and CSL (also known as recombination signal binding protein for immunoglobulin $\kappa \mathrm{J}$ region in mammals). The Notch-1 pathway is abnormally activated in pancreatic cancer cells and its target genes promote several malignant biological behaviors; however, these effects may be suppressed by a Notch-1 inhibitor (si-Notch-1) and DAPT, an inhibitor of $\gamma$-secretase, which is a key molecule that can release NICD from Notch 1 and activate the Notch-1 pathway. Consequently, NE promotes malignant biological behaviors of pancreatic cancer cells in a Notch-1-dependent manner. $\beta 2$-AR, $\beta 2$-adrenergic receptor; MAML, mastermind-like; MMP, matrix metalloproteinase; NICD, Notch intracellular cytoplasmic domain; si, small interfering RNA.

via activating the HPA axis and/or the SNS $(3,4,31)$. As a major downstream factor, NE levels are markedly elevated in the cancer microenvironment $(10 \mu \mathrm{M})$ compared with under normal physiological conditions (10-1,000 pM) $(5,32)$. Furthermore, a clinical trial that contained a large group of patients with cancer demonstrated that $>9,000$ patients were in a state of severe psychological stress and tumor tissues from 30 patients with pancreatic cancer contained a high level of NE $(33,34)$. Furthermore, numerous in vitro and in vivo studies have demonstrated that $\mathrm{NE}$ can induce proliferation, invasion, migration, apoptosis inhibition, angiogenesis and other malignant biological behaviors of PDAC via activation of $\beta 2-\mathrm{AR}$ and its downstream factors, including p38/mitogen-activated protein kinase, cAMP response element binding protein, nuclear factor $-\kappa \mathrm{B}$ and activator protein-1; however, these effects were blocked by $\beta$-receptor antagonists and/or inhibitory neurotransmitter $\gamma$-aminobutyric acid $(6,9,30,35)$. In addition, continuous activation of the Notch-1 pathway has been reported to affect the development of the pancreas and PDAC $(10,19,36,37)$. Previous studies have used transgenic mice to indicate that abnormal activation of the Notch pathway promotes K-RAS oncogene mutations and mediates acinar-to-ductal metaplasia, pancreatic intraepithelial neoplasia and PDAC (19,37-39). In another study, a cyclin-dependent kinase inhibitor dinaciclib (SCH727965) was revealed to suppress the growth of transplanted tumors via inhibiting Notch-1 (40). Furthermore, in vitro studies have reached similar conclusions (41-46). Notably, in previous studies, the Notch-1 pathway was reported to be associated with chronic stress and/or NE in hematopoietic stem cells and breast cancer (20-22). These findings indicated that chronic stress, NE and the Notch-1 pathway may have an interactive relationship in PDAC. Therefore, the present study aimed to elucidate whether NE could promote malignant biological behaviors in PDAC cells via activating the Notch-1 pathway.
The present study initially measured the expression levels of Notch-1 pathway-associated molecules in six pancreatic cancer cell lines; after which one cell line with relatively high Notch-1 expression, BxPC-3, and one with relatively low Notch-1 expression, Panc-1, were selected for further experimentation. Notch-1 and Jagged-1 were revealed to be primarily located on the cytomembrane rather than in the cytoplasm or nucleus of BxPC-3 and Panc-1 cells. Subsequently, PDAC cells underwent a gradient dose/time intervention strategy with NE; the results indicated that NE activated the Notch-1 pathway by increasing the levels of two critical molecules, Notch-1 and Jagged-1. Subsequently, in order to explore whether NE mediated the enhancement of the malignant biological behaviors of PDAC in a Notch-1 pathway-dependent manner, cells were treated with si-Notch-1 or DAPT (Notch-1 pathway inhibitor) to block the Notch-1 pathway during NE treatment. RT-qPCR and western blotting demonstrated that NE affected the expression of Notch-1 pathway-associated genes/proteins in BxPC-3 and Panc-1 cells, and MTT, Annexin V-FITC/PI and Transwell assays revealed that NE enhanced cell viability and invasiveness, and inhibited apoptosis of PDAC cells via activating the Notch-1 pathway. Notably, following inhibition of the Notch-1 pathway using DAPT and si-Notch-1, NE-induced upregulation of Notch-1 pathway target genes, such as Hes-1, MMP-2 and MMP-9, was inhibited. Furthermore, the upregulation of Notch-1 pathway-associated molecules, such as Notch-1, Jagged-1 and RBP-J $\kappa$ was also inhibited. These findings suggested that NE may be an inducer and activator of the Notch-1 pathway; however, the specific mechanism requires further study.

Conversely, si-Notch-1 and DAPT could not completely block NE-mediated progression of PDAC, thus suggesting that other pathways or molecules may influence this process. In addition, the Notch pathway participates in various biological processes and has a complex network of interactions with 
other pathways $(42,43,47)$. Notably, it has been confirmed to serve dual roles in the initiation and progression of several types of cancers or the various stages of a single cancer type $(36,38,48,49)$. Furthermore, NE is just one of the chronic stress hormones, and its use in isolation cannot completely mimic the in vivo chronic psychological stress environment. This is a limitation of the present study, which aimed to explore how chronic stress affects the initiation and progression of PDAC. Therefore, additional studies are required to prove these findings in vivo.

In conclusion, the present study demonstrated that the stress hormone NE may activate the Notch-1 pathway in PDAC and promote its malignant biological behaviors (Fig. 5); this may be an important factor in its development and be associated with a specific underlying mechanism in the progression of PDAC. These findings may provide information regarding a novel approach for targeting NE and/or the Notch-1 pathway in the prevention and treatment of PDAC.

\section{Acknowledgements}

Not applicable.

\section{Funding}

The present study was supported by grants from the National Natural Science Foundation of China (grant nos. 81402971, 81672434 and 81472248).

\section{Availability of data and materials}

The datasets used during the present study are available from the corresponding author upon reasonable request.

\section{Authors' contributions}

WQ, SL, QM and WD designed the experiments. JL performed the majority of the experiments. $\mathrm{KC}, \mathrm{ZJ}$ and $\mathrm{CZ}$ analyzed the data. LC, BY and JC organized the figures and were involved in the conception of the study. WQ wrote the manuscript. QM and WD reviewed the manuscript and supervised this study. All authors read and approved the final manuscript.

\section{Ethics approval and consent to participate}

Not applicable.

\section{Patient consent for publication}

Not applicable.

\section{Competing interests}

The authors declare that they have no competing interests.

\section{References}

1. Siegel RL, Miller KD and Jemal A: Cancer Statistics, 2017. CA Cancer J Clin 67: 7-30, 2017.

2. Kamisawa T, Wood LD, Itoi T and Takaori K: Pancreatic cancer. Lancet 388: 73-85, 2016.
3. Conti CM, Maccauro G and Fulcheri M: Psychological stress and cancer. Int J Immunopathol Pharmacol 24: 1-5, 2011.

4. Clarke DM and Currie KC: Depression, anxiety and their relationship with chronic diseases: A review of the epidemiology, risk and treatment evidence. Med J Aust 190: S54-S60, 2009.

5. Lara HE, Dorfman M, Venegas M, Luza SM, Luna SL, Mayerhofer A, Guimaraes MA, Rosa E, Silva AA and Ramírez VD: Changes in sympathetic nerve activity of the mammalian ovary during a normal estrous cycle and in polycystic ovary syndrome: Studies on norepinephrine release. Microsc Res Tech 59: 495-502, 2002.

6. Quốc Lu'o'ng KV and Nguyễn LT: The roles of beta-adrenergic receptors in tumorigenesis and the possible use of beta-adrenergic blockers for cancer treatment: Possible genetic and cell-signaling mechanisms. Cancer Manag Res 4: 431-445, 2012.

7. Chan C, Lin HJ and Lin J: Stress-associated hormone, norepinephrine, increases proliferation and IL-6 levels of human pancreatic duct epithelial cells and can be inhibited by the dietary agent, sulforaphane. Int J Oncol 33: 415-419, 2008.

8. Guo K, Ma Q, Li J, Wang Z, Shan T, Li W, Xu Q and Xie K: Interaction of the sympathetic nerve with pancreatic cancer cells promotes perineural invasion through the activation of STAT3 signaling. Mol Cancer Ther 12: 264-273, 2013.

9. Huang XY, Wang HC, Yuan Z, Huang J and Zheng Q: Norepinephrine stimulates pancreatic cancer cell proliferation, migration and invasion via $\beta$-adrenergic receptor-dependent activation of P38/MAPK pathway. Hepatogastroenterology 59: 889-893, 2012

10. Gordon WR, Arnett KL and Blacklow SC: The molecular logic of Notch signaling-a structural and biochemical perspective. J Cell Sci 121: 3109-3119, 2008.

11. Gao J, Long B and Wang Z: Role of Notch signaling pathway in pancreatic cancer. Am J Cancer Res 7: 173-186, 2017.

12. Vinson KE, George DC, Fender AW, Bertrand FE and Sigounas G: The Notch pathway in colorectal cancer. Int $\mathbf{J}$ Cancer 138: 1835-1842, 2016.

13. O'Brien R and Marignol L: The Notch-1 receptor in prostate tumorigenesis. Cancer Treat Rev 56: 36-46, 2017.

14. Brzozowa-Zasada M, Piecuch A, Dittfeld A, Mielańczyk $Ł$, Michalski M, Wyrobiec G, Harabin-Słowińska M, Kurek J and Wojnicz R: Notch signalling pathway as an oncogenic factor involved in cancer development. Contemp Oncol 20: 267-272, 2016.

15. Liu J, Shen JX, Wen XF, Guo YX and Zhang GJ: Targeting Notch degradation system provides promise for breast cancer therapeutics. Crit Rev Oncol Hematol 104: 21-29, 2016.

16. Jensen J: Gene regulatory factors in pancreatic development. Dev Dyn 229: 176-200, 2004.

17. Siveke JT, Lubeseder-Martellato C, Lee M, Mazur PK, Nakhai H, Radtke F and Schmid RM: Notch signaling is required for exocrine regeneration after acute pancreatitis. Gastroenterology 134: 544-555, 2008.

18. Miyamoto Y, Maitra A, Ghosh B, Zechner U, Argani P, Iacobuzio-Donahue CA, Sriuranpong V, Iso T, Meszoely IM, Wolfe MS, et al: Notch mediates TGF alpha-induced changes in epithelial differentiation during pancreatic tumorigenesis. Cancer Cell 3: 565-576, 2003.

19. De La O JP, Emerson LL, Goodman JL, Froebe SC, Illum BE, Curtis $A B$ and Murtaugh LC: Notch and Kras reprogram pancreatic acinar cells to ductal intraepithelial neoplasia. Proc Natl Acad Sci USA 105: 18907-18912, 2008.

20. Duncan AW, Rattis FM, DiMascio LN, Congdon KL, Pazianos G, Zhao C, Yoon K, Cook JM, Willert K, Gaiano N, et al: Integration of Notch and Wnt signaling in hematopoietic stem cell maintenance. Nat Immunol 6: 314-322, 2005.

21. Spiegel A, Kalinkovich A, Shivtiel S, Kollet O and Lapidot T: Stem cell regulation via dynamic interactions of the nervous and immune systems with the microenvironment. Cell Stem Cell 3: 484-492, 2008.

22. Chen H, Liu D, Yang Z, Sun L, Deng Q, Yang S, Qian L, Guo L, $\mathrm{Yu} \mathrm{M}, \mathrm{Hu} \mathrm{M}$, et al: Adrenergic signaling promotes angiogenesis through endothelial cell-tumor cell crosstalk. Endocr Relat Cancer 21: 783-795, 2014.

23. Livak KJ and Schmittgen TD: Analysis of relative gene expression data using real-time quantitative PCR and the $2^{-\Delta \Delta C_{\mathrm{T}}}$ method. Methods 25: 402-408, 2001.

24. Duan W, Chen K, Jiang Z, Chen X, Sun L, Li J, Lei J, Xu Q, Ma J, Li X, et al: Desmoplasia suppression by metformin-mediated AMPK activation inhibits pancreatic cancer progression. Cancer Lett 385: 225-233, 2017. 
25. Li X, Ma G, Ma Q, Li W, Liu J, Han L, Duan W, Xu Q, Liu H, Wang Z, et al: Neurotransmitter substance $P$ mediates pancreatic cancer perineural invasion via NK-1R in cancer cells. Mol Cancer Res 11: 294-302, 2013.

26. Liu J, Ma J, Wu Z, Li W, Zhang D, Han L, Wang F, Reindl KM, Wu E and Ma Q: Arginine deiminase augments the chemosensitivity of argininosuccinate synthetase-deficient pancreatic cancer cells to gemcitabine via inhibition of NF- $\kappa$ B signaling. BMC Cancer 14: 686, 2014.

27. Zhang D, Lei J, Ma J, Chen X, Sheng L, Jiang Z, Nan L, Xu Q, Duan $\mathrm{W}$, Wang $\mathrm{Z}$, et al: $\beta 2$-adrenogenic signaling regulates NNK-induced pancreatic cancer progression via upregulation of HIF-1 $\alpha$. Oncotarget 7: 17760-17772, 2016.

28. Weddle DL, Tithoff P, Williams $M$ and Schuller HM: Beta-adrenergic growth regulation of human cancer cell lines derived from pancreatic ductal carcinomas. Carcinogenesis 22 473-479, 2001.

29. Vere CC, Streba CT, Streba LM, Ionescu AG and Sima F: Psychosocial stress and liver disease status. World J Gastroenterol 15: 2980-2986, 2009.

30. Schuller HM, Al-Wadei HA, Ullah MF and Plummer HK III: Regulation of pancreatic cancer by neuropsychological stress responses: A novel target for intervention. Carcinogenesis 33: 191-196, 2012.

31. Herman JP, Figueiredo H, Mueller NK, Ulrich-Lai Y, Ostrander MM, Choi DC and Cullinan WE: Central mechanisms of stress integration: Hierarchical circuitry controlling hypothalamo-pituitary-adrenocortical responsiveness. Front Neuroendocrinol 24: 151-180, 2003.

32. Schmidt $\mathrm{C}$ and Kraft K: Beta-endorphin and catecholamine concentrations during chronic and acute stress in intensive care patients. Eur J Med Res 1: 528-532, 1996.

33. Zabora J, BrintzenhofeSzoc K, Curbow B, Hooker C and Piantadosi S: The prevalence of psychological distress by cancer site. Psychooncology 10: 19-28, 2001.

34. Schuller HM, Al-Wadei HA and Majidi M: GABA B receptor is a novel drug target for pancreatic cancer. Cancer 112: 767-778, 2008

35. Friedman GD, Udaltsova $\mathrm{N}$ and Habel LA: Norepinephrine antagonists and cancer risk. Int J Cancer 128: 737-738, 2011.

36. Dotto GP: Notch tumor suppressor function. Oncogene 27 $5115-5123,2008$

37. Nakhai H, Siveke JT, Klein B, Mendoza-Torres L, Mazur PK, Algül H, Radtke F, Strobl L, Zimber-Strobl U and Schmid RM: Conditional ablation of Notch signaling in pancreatic development. Development 135: 2757-2765, 2008.

38. Mazur PK, Einwächter H, Lee M, Sipos B, Nakhai H, Rad R, Zimber-Strobl U, Strobl LJ, Radtke F, Klöppel G, et al: Notch2 is required for progression of pancreatic intraepithelial neoplasia and development of pancreatic ductal adenocarcinoma. Proc Natl Acad Sci USA 107: 13438-13443, 2010.
39. Hanlon L, Avila JL, Demarest RM, Troutman S, Allen M, Ratti F, Rustgi AK, Stanger BZ, Radtke F, Adsay V, et al: Notch1 functions as a tumor suppressor in a model of K-ras-induced pancreatic ductal adenocarcinoma. Cancer Res 70: 4280-4286, 2010.

40. Feldmann G, Mishra A, Bisht S, Karikari C, Garrido-Laguna I, Rasheed Z, Ottenhof NA, Dadon T, Alvarez H, Fendrich V, et al: Cyclin-dependent kinase inhibitor Dinaciclib (SCH727965) inhibits pancreatic cancer growth and progression in murine xenograft models. Cancer Biol Ther 12: 598-609, 2011.

41. Wang Z, Zhang Y, Banerjee S, Li Y and Sarkar FH: Notch-1 down-regulation by curcumin is associated with the inhibition of cell growth and the induction of apoptosis in pancreatic cancer cells. Cancer 106: 2503-2513, 2006.

42. Gaspar NJ, Li L, Kapoun AM, Medicherla S, Reddy M, Li G, O'Young G, Quon D, Henson M, Damm DL, et al: Inhibition of transforming growth factor beta signaling reduces pancreatic adenocarcinoma growth and invasiveness. Mol Pharmacol 72: 152-161, 2016.

43. Bao B, Wang Z, Ali S, Kong D, Li Y, Ahmad A, Banerjee S, Azmi AS, Miele L and Sarkar FH: Notch-1 induces epithelial-mesenchymal transition consistent with cancer stem cell phenotype in pancreatic cancer cells. Cancer Lett 307: 26-36, 2011.

44. Wang Z, Banerjee S, Li Y, Rahman KM, Zhang Y and Sarkar FH: Down-regulation of notch-1 inhibits invasion by inactivation of nuclear factor-kappaB, vascular endothelial growth factor, and matrix metalloproteinase-9 in pancreatic cancer cells. Cancer Res 66: 2778-2784, 2006

45. Wang Z, Zhang Y, Li Y, Banerjee S, Liao J and Sarkar FH: Down-regulation of Notch-1 contributes to cell growth inhibition and apoptosis in pancreatic cancer cells. Mol Cancer Ther 5: 483-493, 2006

46. Yabuuchi S, Pai SG, Campbell NR, de Wilde RF, De Oliveira E, Korangath P, Streppel MM, Rasheed ZA, Hidalgo M, Maitra A, et al: Notch signaling pathway targeted therapy suppresses tumor progression and metastatic spread in pancreatic cancer. Cancer Lett 335: 41-51, 2013.

47. Wang Z, Sengupta R, Banerjee S, Li Y, Zhang Y, Rahman KM, Aboukameel A, Mohammad R, Majumdar AP, Abbruzzese JL, et al: Epidermal growth factor receptor-related protein inhibits cell growth and invasion in pancreatic cancer. Cancer Res 66: 7653-7660, 2006.

48. Koch U and Radtke F: Notch and cancer: A double-edged sword. Cell Mol Life Sci 64: 2746-2762, 2007.

49. South AP, Cho RJ and Aster JC: The double-edged sword of Notch signaling in cancer. Semin Cell Dev Biol 23: 458-464, 2012. 